University of Wollongong

Research Online

Faculty of Social Sciences - Papers (Archive) Faculty of Arts, Social Sciences \& Humanities

$1-1-2016$

\title{
The spatiotemporal hemodynamic response function for depth-dependent functional imaging of human cortex
}

Alexander M. Puckett

University of Wollongong, apuckett@uow.edu.au

K M. Aquino

University of Sydney

Peter Robinson

University of Sydney, robinson@physics.usyd.edu.au

Michael Breakspear

Queensland Institute of Medical Research, michael.breakspear@qimrberghofer.edu.au

Mark M. Schira

University of Wollongong, mschira@uow.edu.au

Follow this and additional works at: https://ro.uow.edu.au/sspapers

Part of the Education Commons, and the Social and Behavioral Sciences Commons

Research Online is the open access institutional repository for the University of Wollongong. For further information contact the UOW Library: research-pubs@uow.edu.au 


\title{
The spatiotemporal hemodynamic response function for depth-dependent functional imaging of human cortex
}

\begin{abstract}
The gray matter of human cortex is characterized by depth-dependent differences in neuronal activity and connections (Shipp, 2007) as well as in the associated vasculature (Duvernoy et al., 1981). The resolution limit of functional magnetic resonance imaging (fMRI) measurements is now below a millimeter, promising the non-invasive measurement of these properties in awake and behaving humans (Muckli et al., 2015; Olman et al., 2012; Ress et al., 2007). To advance this endeavor, we present a detailed spatiotemporal hemodynamic response function (HRF) reconstructed through the use of high-resolution, submillimeter fMRI. We decomposed the HRF into directions tangential and perpendicular to the cortical surface and found that key spatial properties of the HRF change significantly with depth from the cortical surface. Notably, we found that the spatial spread of the HRF increases linearly from $4.8 \mathrm{~mm}$ at the gray/ white matter boundary to $6.6 \mathrm{~mm}$ near the cortical surface. Using a hemodynamic model, we posit that this effect can be explained by the depth profile of the cortical vasculature, and as such, must be taken into account to properly estimate the underlying neuronal responses at different cortical depths.
\end{abstract}

\section{Keywords}

functional, imaging, function, response, hemodynamic, depth, human, spatiotemporal, cortex, dependent

\section{Disciplines}

Education | Social and Behavioral Sciences

\section{Publication Details}

Puckett, A. M., Aquino, K. M., Robinson, P. A., Breakspear, M. \& Schira, M. M. (2016). The spatiotemporal hemodynamic response function for depth-dependent functional imaging of human cortex. Neurolmage, 139 (October), 240-248. 
1 Title: The spatiotemporal hemodynamic response function for depth-dependent

2 functional imaging of human cortex

3 Abbreviated title: The spatiotemporal hemodynamic response function

4 Authors/Affiliations:

5 Alexander M. Puckett ${ }^{a}$, Kevin M. Aquino ${ }^{b, c, d, e}$, P. A. Robinson ${ }^{b, d}$, Michael

6 Breakspear ${ }^{\mathrm{c}, \mathrm{f}}$, Mark M. Schira ${ }^{\mathrm{a}, \mathrm{g}}$

7 a School of Psychology, University of Wollongong; Wollongong NSW 2522;

8 Australia

9 bSchool of Physics, University of Sydney; Sydney NSW 2006; Australia

$10{ }^{\circ}$ Center for Integrative Brain Function, QIMR Berghofer Medical Research

11 Institute; Herston QLD 4006; Australia

$12{ }^{\mathrm{d} C e n t e r}$ for Integrative Brain Function, University of Sydney; Sydney NSW

13 2006; Australia

$14{ }^{\text {e}}$ Sir Peter Mansfield Imaging Center; Nottingham, Nottinghamshire, NG7 2RD;

15 United Kingdom

16 'Metro North Mental Health Service, Royal Brisbane and Women's Hospital;

17 Herston QLD 4029; Australia

18 9Neuroscience Research Australia; Randwick NSW 2031; Australia

19 Corresponding author (with present address):

20 Alexander Puckett

21 Queensland Brain Institute, University of Queensland; Brisbane QLD 4072;

22 Australia

23 pucketta@alumni.msoe.edu 


\section{Abstract}

25 The gray matter of human cortex is characterized by depth-dependent

26 differences in neuronal activity and connections (Ship, 2007) as well as in the

27 associated vasculature (Duvernoy et al., 1981). The resolution limit of functional

28 magnetic resonance imaging (fMRI) measurements is now below a millimeter,

29 promising the non-invasive measurement of these properties in awake and

30 behaving humans (Muckli et al., 2015; Olman et al., 2012; Ress et al., 2007). To

31 advance this endeavor, we present a detailed spatiotemporal hemodynamic

32 response function (HRF) reconstructed through the use of high-resolution,

33 submillimeter fMRI. We decomposed the HRF into directions tangential and

34 perpendicular to the cortical surface and found that key spatial properties of the

35 HRF change significantly with depth from the cortical surface. Notably, we found

36 that the spatial spread of the HRF increases linearly from $4.8 \mathrm{~mm}$ at the

37 gray/white matter boundary to $6.6 \mathrm{~mm}$ near the cortical surface. Using a

38 hemodynamic model, we posit that this effect can be explained by the depth

39 profile of the cortical vasculature, and as such, must be taken into account to

40 properly estimate the underlying neuronal responses at different cortical depths.

\section{$42 \quad$ Keywords}

43 High-resolution, fMRI, hemodynamic response, human, visual cortex, layers 


\section{1. Introduction}

45 Advances in imaging hardware and analysis techniques have pushed the

46 resolution limits of $\mathrm{fMRI}$ below a millimeter, allowing blood oxygenation level

47 dependent (BOLD) responses to be resolved through the depth of cortical gray

48 matter (Jin \& Kim, 2008; Siero et al., 2011; Silva et al., 2000). In parallel with

49 advances in modeling and deconvolution techniques (Aquino et al., 2014;

50 Drysdale et al., 2010), this delivers the potential to infer depth-dependent

51 neuronal activity and to thereby distinguish feedforward, feedback, and recurrent

52 neuronal activity in cortical circuits (Bastos et al., 2012; Logothetis, 2008).

53 However, this requires a characterization of the spatiotemporal structure present

54 in the BOLD response and specifically how it changes through cortical depth.

56 A number of models have been used to describe the hemodynamic response

57 that the BOLD signal represents, and these models have grown in complexity

58 with our understanding of the response. Early on the HRF was described using

59 models that only characterized the temporal dimension (Buxton et al., 1998;

60 Friston et al., 1994). More recently, work has been done to incorporate both the

61 temporal and spatial aspects of the BOLD response giving way to HRF models

62 that describe the spatial aspect of the response tangential to the cortical surface,

63 the temporal dimension, and how these two components relate (Aquino et al.,

64 2012; Shmuel et al., 2007). Other work has described changes in various

65 aspects of the HRF through the depth of cortex (Koopmans et al., 2010; Olman

66 et al., 2012; Ress et al., 2007; Siero et al., 2015; Yu et al., 2012), and Heinzle et 
67 al. (2016) have recently extended a commonly used hemodynamic model

68 (Buxton et al., 1998; Friston et al., 2000; Stephan et al., 2007) to incorporate

69 blood draining effects across cortical depth to explain some of these differences.

70 To date, however, there is no characterization that features the complete

71 spatiotemporal structure of the BOLD response, that is both tangential and

72 perpendicular to the cortical surface.

74 Here we measure the HRF in primary visual cortex using high-resolution fMRI

75 and quantify how the response changes in time and space, both tangential to the

76 cortical surface and through the depth of cortex. This constitutes the first

77 characterization of the spatiotemporal HRF that can be used for depth-dependent

78 functional imaging of human cortex.

80 We also relate our empirical measurements to a recent physiologically-based

81 model of the HRF (Drysdale et al., 2010) that approximates cortical tissue as a

82 porous elastic medium enabling the hemodynamics to be explained in a

83 continuous mean-field manner. An important finding from this work was that

84 waves of hemodynamic activity were predicted and supported by results in

85 primary visual cortex (Aquino et al., 2012). These waves couple space and time,

86 supporting the notion that a complete description of the BOLD response requires

87 a spatiotemporal model of hemodynamics (Biessman et al., 2012; Gardner et al.,

88 2010; Kriegeskorte et al., 2010; Shmuel et al., 2007). The hemodynamic waves

89 travel along the cortical sheet and are characterized by two parameters: 
90 hemodynamic velocity and damping rate, both of which reflect basic properties of

91 the underlying physiology (Aquino et al., 2012). Here we find further evidence of

92 these waves and examine how their properties change as a function of cortical 93 depth.

94

95 2. Materials and Methods

962.1 Overview

97 To measure the spatiotemporal structure of the HRF we combined sub-millimeter

$98 \mathrm{MRI}$ with an experimental design to maximize the spatial and temporal resolution 99 of our data $(0.8 \mathrm{~mm}$ isotropic resolution, $500 \mathrm{~ms}$ effective sampling rate).

100 Exploiting the organization of primary visual cortex (Schira et al., 2010) we used 101 a visual stimulus (Fig. 1A) that elicits a thin line of cortical activation stretching 102 across $\mathrm{V} 1$ (Fig. 1B). To investigate the spatiotemporal structure of the HRF 103 tangential to the cortical surface we sampled the temporal $f M R I$ response as a 104 function of the distance from the center of activation (Fig. 1C). To quantify how 105 the response varies perpendicular to the surface this was done at a series of 106 different cortical depths. For these last two steps, we implemented a custom, 107 surface-based sampling method. 
A.

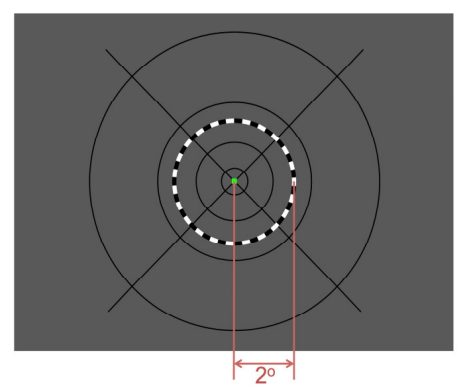

B.

\subsection{Subjects} guidelines.
C.

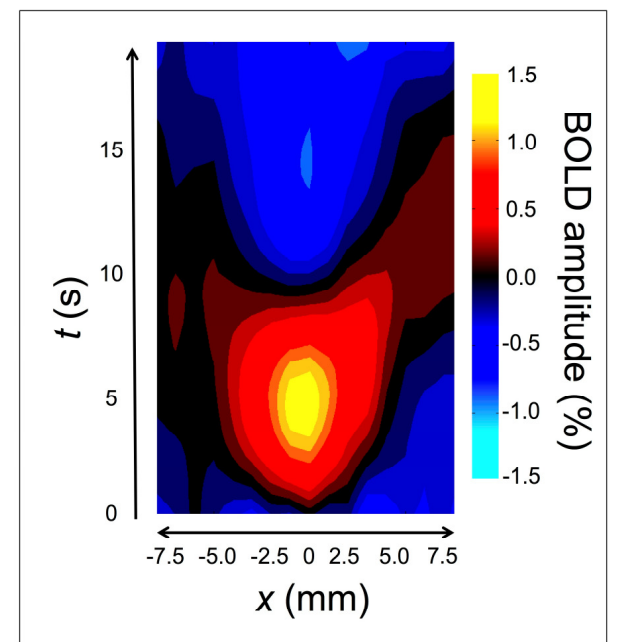

Figure 1. Measuring the spatiotemporal HRF. A. The visual stimulus was a thin, black and white, flickering ring designed to stimulate isoeccentric responses in visual cortex. B. Medial view of a computationally inflated cortical surface mesh. Zoomed region shows primary visual cortex, V1. Distance from the center of activation elicited by the stimulus is color-coded. C. Spatiotemporal HRF plot illustrating the structure of the HRF parallel to the surface. $x$ is the distance from the center of activation, where positive is toward the periphery and negative is toward the fovea. The color indicates BOLD signal amplitude (\%).

Six subjects (2 female; 22-35 years, mean 27 years) with normal or corrected-tonormal vision and no history of neurological or psychiatric diseases participated.

The experiment was conducted with the written consent of each subject and was approved by the Human Ethics Committee at the University of Queensland in accordance with the Australian National Health and Medical Research Council's 
127 MRI scanning was performed using a Siemens MAGNETOM Trio 3T MRI 128 scanner with a 32-channel head coil at the Centre for Advanced Imaging at the

129 University of Queensland. Multiple T1-weighted anatomical images were 130 collected: (1) full brain MPRAGE with $0.75 \mathrm{~mm}^{3}$ resolution using a TE of $2.7 \mathrm{~ms}$,

131 TR of $1900 \mathrm{~ms}, 9$ degree flip angle, FOV of $240 \mathrm{~mm}$, matrix size of $320 \times 320$,

132 and 224 slices; (2) partial coverage MPRAGE focusing on the occipital pole with

$1330.50 \mathrm{~mm}^{3}$ resolution using a TE of $5.0 \mathrm{~ms}$, TR of $2410 \mathrm{~ms}, 9$ degree flip angle, 134 FOV $210 \mathrm{~mm}$, matrix size of $416 \times 416$, and 256 slices; (3) partial coverage 135 MP2RAGE focusing on the occipital pole with $0.54 \mathrm{~mm}^{3}$ resolution using a TE of $1363.0 \mathrm{~ms}$, TR of $4000 \mathrm{~ms}$, flip angles of 6 and 7 degrees, FOV $210 \mathrm{~mm}$, matrix size 137 of $390 \times 390$, and 256 slices. Each of the anatomical datasets were subsequently 138 aligned through a rigid body transform with 6 degrees of freedom using SPM8 139 (http://www.fil.ion.ucl.ac.uk/spm/software/spm8/). All images were interpolated to $1400.4 \mathrm{~mm}^{3}$ and then averaged to construct a high quality, high-resolution 141 anatomical image for each subject. The lower resolution sequence $\left(0.75 \mathrm{~mm}^{3}\right)$ 142 provided high SNR and coverage of the entire brain while the two different higher 143 resolution protocols, although noisier, provided better definition of the gray/white 144 boundary at the occipital pole. The high-resolution of this anatomy and hence 145 surface reconstruction was particularly helpful with estimation of distances along 146 the cortical surface.

148 All functional data were acquired using a gradient-echo sequence with a matrix 
149 size of $240 \times 240$ and an FOV of $192 \mathrm{~mm}$, resulting in an in-plane resolution of

$1500.8 \mathrm{~mm} \times 0.8 \mathrm{~mm}$. Within a TR of $4 \mathrm{~s}, 37$ slices $(0.8 \mathrm{~mm}$ thick) were acquired

151 using a flip angle of 90 degrees, a TE of $38 \mathrm{~ms}$, an echo spacing of $1.31 \mathrm{~ms}$, an

152 EPI factor of 60 , Generalized Autocalibrating Partially Parallel Acquisition

153 (GRAPPA) (Griswold et al., 2002) with an acceleration factor of 3, an effective

154 bandwidth of $768 \mathrm{kHz}$, and 6/8 partial Fourier sampling. This results in a readout

155 length of $78.6 \mathrm{~ms}(60 \times 1.31 \mathrm{~ms})$, which increases the voxel size in the phase-

156 encode direction by approximately $15-17 \%$ using a gray matter T2* of $40 \mathrm{~ms}$ at $3 \mathrm{~T}$

157 (Haacke et al., 1999). The acquisition slab was positioned at the edge of the at

158 the occipital pole and extended anteriorly ensuring coverage of V1.

160 Functional data were slice time corrected using SPM8. Motion correction was

161 performed using a two-step procedure in which head motion vectors were first

162 estimated using SPM8. These vectors were then low-pass filtered (cut-off 45

163 seconds) and then applied to the EPI volume time series. This prevented strong

164 BOLD signal modulations present in the high-resolution EPI data from affecting

165 the motion correction (Schira et al., 2010). Using custom MATLAB scripts and

166 the mrVista Toolbox (http://white.stanford.edu/software/), the data were aligned

167 and interpolated (trilinear) to match the space defined by the anatomical data

168 (see Inline Supplementary Fig. S1). The time series for each voxel was low-pass

169 filtered using a third order Butterworth filter with a frequency cutoff at $0.1 \mathrm{~Hz}$. 
172 Visual stimulation was presented on a white screen using an LCD projector and

173 viewed using a mirror mounted on the head coil. The display extended to $15^{\circ}$

174 eccentricity. The stimulus was designed and presented using Psychtoolbox

175 version 3 (Brainard, 1997; Pelli, 1997).

176

177 The stimulus used (Fig. 1A) consisted of a very thin (1 pixel), black and white

178 flickering (250 ms contrast reversal) ring stimulus with a radius of 2 degrees

179 ('ON' for 4 seconds) that alternated with a uniform gray field ('OFF' for $16.5 \mathrm{~s}$ ).

180 The stimulus timing was designed to elicit robust BOLD response while avoiding

181 nonlinearities in the BOLD signal known to be elicited by very brief ON periods

182 (less than 2-3 $\mathrm{s}$ in visual cortex) and OFF periods (less than $4 \mathrm{~s}$ ) (Birn \&

183 Bandettini, 2005; Soltysik et al., 2004). The stimulus was designed to induce a

184 discrete isoeccentric band of neural responses in visual cortex approximately 1

$185 \mathrm{~mm}$ in width (Tootell et al., 1981; Vanduffel et al., 2002) and is similar to that

186 used in our previous study (Aquino et al., 2012). Subjects performed a fixation

187 color detection task at a central maker, and a fixation grid was overlaid on the

188 stimulus to stabilize fixation throughout the entire run. Previous studies have

189 demonstrated that with this stimulus setup, subjects typically fixate very

190 accurately, within 10' of visual angle, probably better (Schira et al., 2007). This

191 fixation jitter results in a spread of the representation of the ring of less than 0.54

$192 \mathrm{~mm}$ on the cortical surface (Schira et al., 2010). The ON/OFF cycle (totaling 20.5

193 s) was repeated 8 times per run thereby allowing the evoked response to be 
194 sampled with an effective temporal resolution higher than the scanner repetition

195 time. This was achieved by the stimulus onset being dephased by $500 \mathrm{~ms}$ per

196 cycle for 8 cycles, and then reordering the time-course to acquire a single

197 response at an effective temporal resolution of $500 \mathrm{~ms}$. Each run was 3 minutes

198 long and 18-25 runs were collected across two MRI sessions for each subject.

200 In addition to the thin ring stimuli, phase-encoded retinotopic mapping was

201 performed to acquire polar angle maps for visual area identification. A rotating

202 bowtie stimulus (two wedges opposite one another and meeting at fixation) was

203 used (Schira et al., 2009). The aperture contained one of three colored texture

204 patterns (checkers, expanding and contracting spirals, or rotating sinusoidal

205 gratings) which changed randomly every 250 ms. Each retinotopic mapping run

206 was 6 minutes in duration (15 cycles), and two runs were collected per subject.

207 Subjects performed a fixation color detection task at a central maker, and a

208 fixation grid was overlaid atop the stimuli.

209

$210 \quad 2.5$ Laminar surface reconstruction and data sampling

211 To characterize the functional data at different cortical depths, a surface-based

212 sampling method was used. First, a detailed, manual gray/white matter

213 segmentation of the average anatomical image was performed for each subject

214 using the segmentation program ITK-Gray (Yushkevich et al., 2006). Based on

215 this segmentation, high-resolution cortical surface meshes (approximately 0.4

$216 \mathrm{~mm}$ between nodes) were constructed using the cortical surface analysis 
217 package Caret, version 5.65 (Van Essen et al., 2001). Caret was chosen as it

218 allows the construction of precise parallel surfaces shrunk (into white matter) or

219 expanded (toward the pial surface) along the surface normals (i.e., in the

220 direction that is perpendicular to the surface at each vertex). Note that this

221 procedure ensures that all laminar surface meshes contain the same number of

222 nodes and edges. In total, 6 surfaces were constructed for each subject located -

$2230.8,0,0.8,1.6,2.4$, and $3.2 \mathrm{~mm}$ from the gray/white matter boundary (negative is

224 into white matter, positive is towards the cortical surface). The volumetric fMRI

225 data were then manually aligned to the anatomical data (using the mrVista

226 software) and subsequently linearly interpolated onto each of these surfaces

227 using MATLAB. Primary visual cortex is rather thin with a thickness of

228 approximately $2.0-2.5 \mathrm{~mm}$ (Scholtens et al., 2015; Wagstyl et al., 2015);

229 therefore, the surface constructed at $3.2 \mathrm{~mm}$ from the gray/white boundary will be

230 primarily sampling data from the large surface vessels located outside the pial

231 surface (vascular arachnoid).

232

\section{$233 \quad 2.6$ Region of interest and distance measurements}

234 The analysis was restricted to primary visual cortex, V1, as determined by the

235 polar angle retinotopy data (DeYoe et al., 1996; Wandell \& Winawer, 2011). The

236 centerline of the activation elicited by the thin ring stimulus was identified as

237 described previously (Figure 4 in Aquino et al., 2012). Briefly this is achieved by

238 (i) identifying clusters of voxels that correlate to the task on a flattened

239 representation of $\mathrm{V} 1$, (ii) fitting a polynomial to this cluster, and (ii) selecting 
240 voxels close to this polynomial to represent the centerline. For each laminar

241 surface, all further analyses were restricted to only those surface nodes within 15

$242 \mathrm{~mm}$ of the centerline (in both directions). The geodesic distance of the shortest

243 path between each surrounding mesh node and the centerline was calculated

244 using a fast-marching algorithm (Sethian, 1999) implemented in MATLAB. By

245 allowing the shortest path to cross mesh faces rather than being bound to the

246 edges, the fast-marching algorithm avoids metrication error inherent in other

247 techniques such as Dijkstra's algorithm (Bronstein et al., 2008). The distance

248 measures were used to investigate how the hemodynamic response changes

249 with distance from the source of activation, tangential to the cortical surface. The

250 distances between surface nodes change with cortical depth; therefore, the

251 distance measures were calculated for each laminar surface individually.

\subsection{Spatiotemporal HRF profile}

254 Spatiotemporal HRF responses were constructed by averaging time-course 255 responses based on the distance of the responses from the centerline of 256 activation, using a $1 \mathrm{~mm}$ bin size. Each surface node is approximately, but not 257 exactly, $0.4 \mathrm{~mm}$ from one another. Hence using a $1 \mathrm{~mm}$ bin size ensured the 258 distance bin containing the centerline of activation was comprised of the 259 centerline node and at least one surface node in each direction away from the 260 centerline. These averaged responses were then used to compute a contour plot 261 (Fig. 1C) that shows the structure of the HRF tangential to the cortical surface in 262 which distance from the centerline is represented along the $\mathrm{x}$-axis (negative 
263 refers to distance towards the fovea, that is roughly towards the posterior of the

264 brain and positive signifies distance toward the periphery of the retinotopic map,

265 that is roughly anterior), time is represented along the y-axis, and color

266 represents BOLD amplitude. Note that the information included in a typical,

267 temporal HRF profile is represented in our spatiotemporal profile. Rather than

268 represent time on the $\mathrm{x}$-axis and amplitude on the y-axis, time is represented on

269 the $y$-axis and amplitude as color (see Inline Supplementary Fig. S2). The spatial

270 range (i.e., the $\mathrm{x}$-axis limits) was chosen to exclude interference from other

271 sources. Individual HRF profile plots were constructed for each laminar surface

272 (e.g. Fig. 2, right). Group-averaged spatiotemporal HRF profile plots were

273 constructed (Fig. 2, left) at each cortical depth by averaging the HRF profiles

274 across all 12 hemispheres. The averaging was achieved by calculating the mean

275 time-course within each distance bin across the profiles from the individual 276 subjects.

277

278 To estimate the spatial spread and peak amplitude of the HRF, a Gaussian

279 function was fitted to the spatial component, $s(x)$, of the peak response in each

280 hemisphere with:

281

282

$$
s(x)=A \exp \left[-\frac{\left(x-x_{0}\right)^{2}}{\Delta x^{2} /(2 \sqrt{\ln 2})}\right]
$$

284 where $A$ is the peak signal amplitude, $x_{0}$ is the spatial location of the peak of 285 activation, and $\Delta x$ is the full-width-at-half-maximum (FWHM) of the Gaussian 
286 function. As the time to peak varied slightly across subjects, the response peaks

287 between 5-6 s following stimulus onset were used. The parameters were fitted

288 using the Trust-Region algorithm implemented in MATLAB's fit function.

289

\subsection{Estimation of hemodynamic wave parameters}

291 The hemodynamic response can be represented as a planar wave (i.e., a wave 292 traveling through space $x$ and time $t$ ):

293

$294 S(x, t)=\operatorname{Re}\left[e^{-i k x-t(\Gamma \pm i k v)}\right]$

295

296 where $\mathrm{S}$ is the signal, $\mathrm{k}$ is the wave number, $v$ is the wave velocity, and $\Gamma$ is the

297 wave damping parameter (Aquino et al., 2014). Note that the \pm indicates waves

298 traveling towards the positive and negative directions.

300 We calculated the wave parameters $\Gamma$ and $v$ for the group-averaged

301 hemodynamic responses at different depths as previously described (Aquino et 302 al., 2012). Briefly, to calculate $v$ we fitted a line to the instantaneous signal phase 303 fronts traveling from $x=0$ outwards at the peak of the BOLD amplitude. As 304 described in Aquino et al. (2014) the predicted hemodynamic response contains 305 a local mode which is a temporal mode that is non-propagating, and a 306 propagating mode which encompasses the propagation due to hemodynamic 307 waves. Thus to ensure we capture the propagating mode only, we calculated 308 these wave fronts outside $2 \mathrm{~mm}$ from the center (central core), which ensures 
309 that the local mode is not contributing to the hemodynamic response.

310 Additionally, we restricted the velocity calculation to before the edge to ensure

311 we capture the waves and not additional sources. The instantaneous signal

312 phase was calculated using a Hilbert transform. To calculate $\Gamma$, we measured the

313 exponent of exponential signal decay along the signal wave front by linear 314 regression.

316 There is some variation in the wave parameters left and right of the stimulus

317 (towards and away from the fovea, respectively). It is likely that this arises from

318 changes in vascular density that follow changes in neural density as a function of

319 eccentricity. Here we have, however, restricted our analysis to the the average

320 between these two quantities as additional data is required to properly

321 investigate this variation.

\subsection{Statistical analyses}

324 Two levels of statistics were implemented in IBM SPSS Statistics 19.0. First, two

325 separate repeated measures ANOVA tests were performed to test for statistically

326 significant differences across cortical depth for both the spatial spread and peak

327 amplitude measurements. These were performed using the measurements

328 acquired from the Gaussian fitting procedure in each individual hemisphere $(\mathrm{n}=$

329 12). Mauchly's test revealed that the assumption of sphericity underlying both

330 repeated measures ANOVAs was violated so Greenhouse-Geisser corrections

331 were used. Because both the spatial spread and the amplitude of the HRF were 
332 found to vary significantly with depth $[F(1.86,20.5)=5.61, p=0.013$ and

$333 F(1.22,13.4)=9.75, p=0.006$ respectively], a second level of statistics was

334 performed to investigate the relationships between each dependent variable

335 (spatial spread and amplitude) and cortical depth using linear regression. The

336 uncertainty values $( \pm)$ associated with the reported parameter estimates

337 (coefficients) indicate standard error.

\section{3. Results}

340 Surface reconstruction of the BOLD response yielded a spatiotemporal HRF,

341 describing the response across (1) time, (2) space tangential to the cortical

342 surface, and (3) space perpendicular to the cortical surface, through cortical

343 depth. The response displays a number of notable properties that depend on

344 cortical depth (Fig. 2). As expected, there is very little response modulation at $z=$

$345-0.8 \mathrm{~mm}$ (that is $0.8 \mathrm{~mm}$ below the gray/white boundary; Fig. 2, bottom panel). At

346 more superficial depths (increasing $z$ ) the response is stronger and broader.

347 Moreover, at all depths much of the response propagates as a traveling wave,

348 evident as increasingly delayed BOLD changes away from the central response.

349 Note that in Figure 2 the spatiotemporal HRF is illustrated by a series of plots

350 showing the response tangential to the surface vs. time with each plot in the

351 series representing data at a different cortical depth. The same data is provided

352 in an alternate video format showing the response tangential to the surface vs.

353 space orthogonal to the surface with each video frame representing the response

354 at a different time point (see Supplementary Material, S3 and S4). 

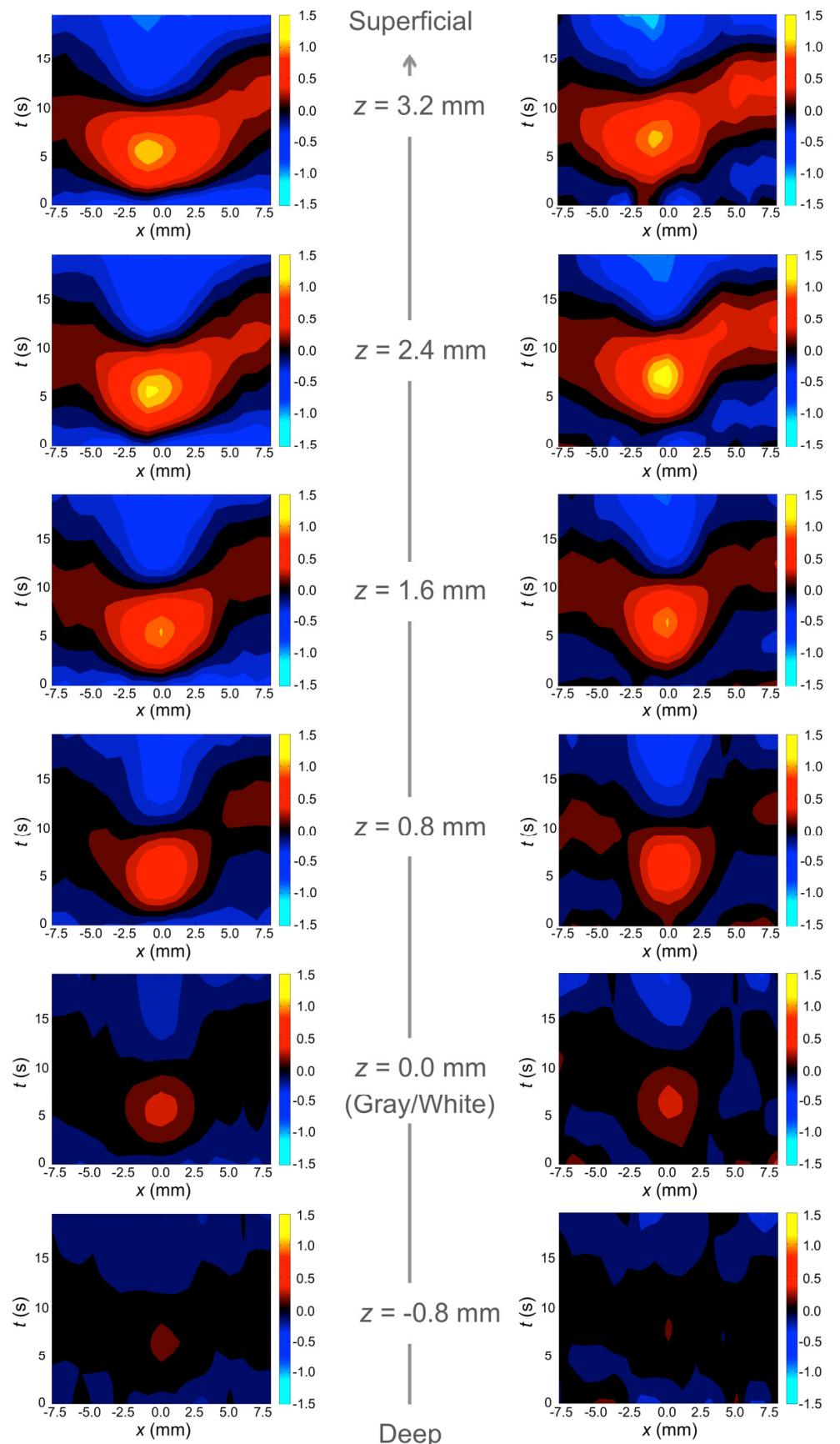

356 Figure 2. Group-averaged (left) and single subject (right) spatiotemporal HRF 357 plots vs. cortical depth relative to the gray/white boundary, $z$ (middle). Each plot 358 represents a slice through the HRF at a different cortical depth and illustrates the 359 structure of the HRF tangential to the surface at that depth. Color indicates the percent modulation of BOLD. 
363 The spatiotemporal hemodynamic response thus varies through the depth of 364 gray matter. We quantified the spatial spread $\Delta x$ and the peak amplitude $A$ of the 365 spatiotemporal HRF as a function of $z$ by fitting a Gaussian to each individual 366 spatial profile at the peak time point 5-6 seconds following the onset of the BOLD 367 response (Fig. 3, top). This analysis confirmed that both $\Delta x$ and $A$ vary 368 significantly with $z(p<0.05)$, quantifying the visually apparent effect. Moreover, 369 the variations in $\Delta x$ and $A$ are well described by linear regression (both $p \leq$ $370 \quad 0.001)$ with

371

372

$$
\Delta x=(0.6 \pm 0.2)^{*} z+(4.8 \pm 0.4) \mathrm{mm}
$$

$R^{2}=0.2$,

373 and

374

$$
A=\left[(0.30 \pm 0.07) \% \mathrm{~mm}^{-1}\right]^{\star} z+(0.5 \pm 0.1) \%
$$$$
\mathrm{R}^{2}=0.2
$$ 


\section{HRF Properties Across Cortical Depth}
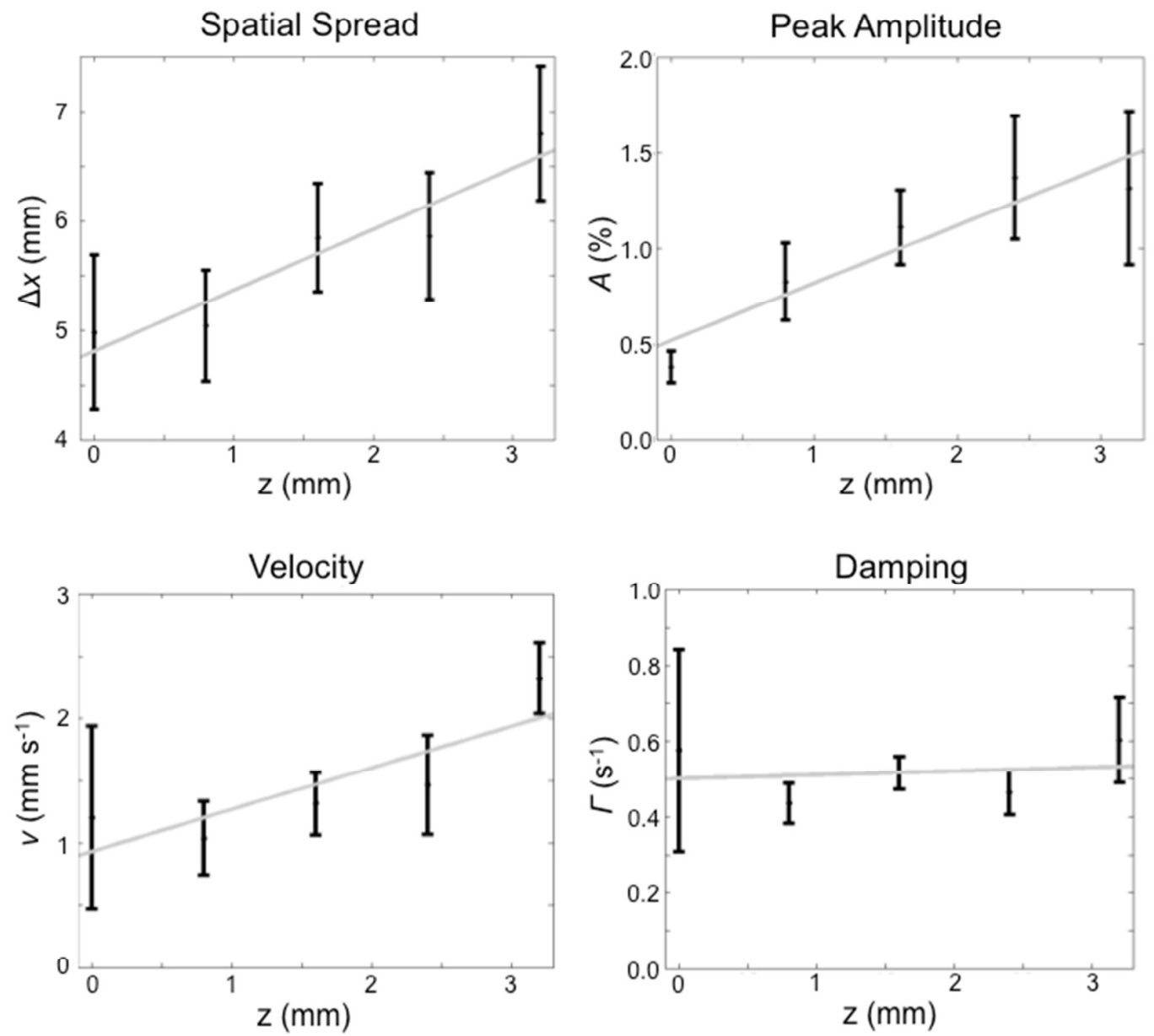

377 Figure 3. HRF properties vs. $z$. Top: Variation in the spatial spread $\Delta x$ and peak amplitude $A$ of the HRF vs. $z$. Depth at $z=0$ corresponds to the gray/white boundary. Increasing $z$ moves towards the pial surface. Error bars correspond to standard error (across 12 hemispheres). Solid gray lines show the linear regression fits. Bottom: Wave parameter estimates $v$ and $\Gamma$ calculated for the group-averaged hemodynamic response as a function of $z$. The points indicate the average of each parameter calculated for the wave fronts towards the fovea and towards the periphery. Error bars indicate the standard error across profiles, and the solid gray lines show the linear regression fits. 


\section{$387 \quad 3.2$ Hemodynamic traveling waves and the underlying physiology}

388 The spatiotemporal HRF plots in Figure 2 show clear evidence of traveling waves 389 at all depths, consistent with the predictions of our recent, physiologically-derived 390 model (Aquino et al., 2012). This is not only true for the average response (Fig. 3912 2, left) but can also be seen in the responses of individual subjects (cf. Fig. 2, 392 right). The hemodynamic waves can be characterized by two key parameters: 393 the hemodynamic velocity $v$, which corresponds to the speed of the response 394 propagation, and the wave damping $\Gamma$, which describes the signal decay. In the 395 hemodynamic model, these parameters reflect basic properties of the physiology. 396 The velocity $v$ depends on the average stiffness of cortical vasculature, while 397 damping $\Gamma$ is attributed to blood viscosity and blood outflow. Note that in the 398 mean field, these are averaged quantities and the velocity parameter also 399 depends on the mean resistance of vessels as encapsulated by the dependence 400 on resting pressure.

401

402 We estimated $v$ and $\Gamma$ at each cortical depth (Fig. 3, bottom) using a linear fit and 403 found that velocity $v$ shows a trend level increase with $z(p=0.07 \mathrm{~F}$-test, using a 404 linear model vs a constant model) while damping $\Gamma$ is uniform vs. $z$ ( $p=0.77 \mathrm{~F}$ 405 test, using a linear model vs a constant model). The results were restricted to $z>$ $4060(z=-0.8 \mathrm{~mm}$ was high in signal noise) and are shown in Figure 3 (bottom). The 407 regression equation for $v$ is given by, 
411 and for $\Gamma$,

415 where the uncertainty values $( \pm)$ indicate standard error of the estimated 416 parameters.

\section{Discussion}

419 We found that the amplitude $A$ and spatial spread $\Delta x$ of the HRF decrease with 420 depth (i.e., smaller values of $z$ ), and this can be approximated as a simple linear 421 decrease for both amplitude and spatial spread. We also found that the 422 hemodynamic wave velocity $v$ increases towards the surface while damping $\Gamma$ 423 remains uniform through cortical depth. In wave theory these parameters 424 determine the spatial extent of the hemodynamic waves, estimated by the 425 characteristic range $R$ (Aquino et al., 2012) given by

$$
R=v / \Gamma
$$

429 and taking the approximation that $\Gamma$ is roughly constant, the characteristic range 430 for the hemodynamic waves is

$$
\mathrm{R} \sim(0.6 \pm 0.2) \mathrm{z}+(1.8 \pm 0.4) \mathrm{mm}
$$


432 where it is noted that these waves are in addition to the central core of activation.

433 Therefore, our finding that $R$ increases with $z$ suggests that the observed 434 increase in the spatial spread of the HRF $(\Delta x)$ toward the surface reflects depth435 dependent differences in vascular properties. Specifically, $v$ is dependent on the

436 linear elasticity coefficient of cortical vessels; hence the observed changes imply 437 an increase in the linear elasticity coefficient as a function of $z$. This finding is 438 consistent with prior knowledge that vessels closer to the pia have more elastic 439 tissue (ladecola, 2004; Zhang et al., 1990). In particular, recent work in optical 440 imaging and modeling found that the dilatory expressions of cortical vessels 441 increase from the gray/white boundary to the surface owing to the corresponding 442 increases in the Virchow-Robin space (Gao et al., 2015).

444 In agreement with our findings, it has been shown by a number of groups that the 445 amplitude of the BOLD response increases toward the cortical surface (Chen et 446 al., 2013; Koopmans et al., 2010; Ress et al., 2007). In addition to the increase in 447 amplitude, Polimeni et al. (2010) demonstrated a loss in spatial specificity of the 448 BOLD response towards the surface. Importantly, our experimental design 449 permitted to describe, quantify, and explain this in terms of depth-dependent 450 changes in the spatial spread of the HRF. In combination with our physiological 451 model this suggests that the loss of specificity is not the result of large surface 452 vessels alone, but rather reflects a more gradual change in vascular elasticity 453 that appears to occur monotonically with depth. 


\subsection{Implications for depth-dependent fMRI}

456 It has long been acknowledged that neuronal modulation does not only change

457 the BOLD signal in the immediate voxel surrounding the response, but that the

458 modulation extends in space to neighboring voxels (Engel et al., 1997). Despite

459 this, the HRF is typically characterized only in the temporal dimension, with some

460 analyses completely ignoring the spatial component of the response (Boynton et

461 al., 2012). Furthermore, analysis methods that do consider both the temporal and

462 spatial aspects of the HRF regard the HRF as the product of a spatial and

463 temporal component. This implicitly enforces the approximation that the spatial

464 and temporal dynamics of the hemodynamic response are uncoupled.

466 Due to the range of these effects, traditional $\mathrm{fMRI}$ studies (e.g. region based-

467 studies with resolution $>=3 \mathrm{~mm}$ ) are less affected; however, the assumption that 468 the spatial and temporal dynamics of the hemodynamic response are uncoupled 469 is untenable in the face of high spatial resolution fMRI. We have previously 470 shown that this approximation is unphysical as there is an intrinsic 471 spatiotemporal coupling in the hemodynamic response tangential to the cortical 472 surface (Aquino et al., 2012). This introduces interdependence in the BOLD 473 response among neighboring voxels in a time-dependent manner (Biessmann et 474 al., 2012; Kriegeskorte et al., 2010; Shmuel et al., 2007). We have also shown 475 that accounting for this interdependence using a spatiotemporal HRF model and 476 an integrated spatial and temporal deconvolution results in improved estimation 477 of the underlying neuronal responses compared to a more conventional analysis 
478 using a space-time separable HRF (Aquino et al., 2014), even for medium 479 resolution data (such as $1.5 \mathrm{~mm}^{3}$ voxels).

480

481 In order to apply spatiotemporal deconvolution to estimate depth-dependent 482 neuronal responses, it is vital to determine the hemodynamic changes at different 483 cortical depths. Here, we extend our previous work by examining the 484 spatiotemporal structure of the hemodynamic response perpendicular to the 485 cortical surface, quantifying key changes in the HRF with cortical depth. The 486 magnitudes of these changes are such that they must be accounted for in order 487 to properly interpret depth-dependent BOLD measurements. For example, the 488 spatial spread of the hemodynamic response tangential to the surface varies by 489 nearly $2 \mathrm{~mm}$ (equivalent to 2.5 voxels at the resolution employed here) through 490 the thickness of the cortical sheet.

492 Our present observations coupled with our past work therefore demonstrate the 493 necessity and enable the use of a spatiotemporal HRF model for depth494 dependent fMRI of human cortex. The empirically derived velocity, damping, and 495 amplitude estimates (Fig. 3) in combination with the theoretically derived 496 framework of the poroelastic model of hemodynamics (Aquino et al 2014; 497 Drysdale et al., 2010) provide such a spatiotemporal HRF model (Fig. 4). 498 Moreover, we suggest that just as deconvolution is now standard for diffusion 499 (Tournier et al., 2004) and susceptibility imaging (Li et al., 2012), its use (with a 500 spatiotemporal HRF model) should become standard for fMRI. 


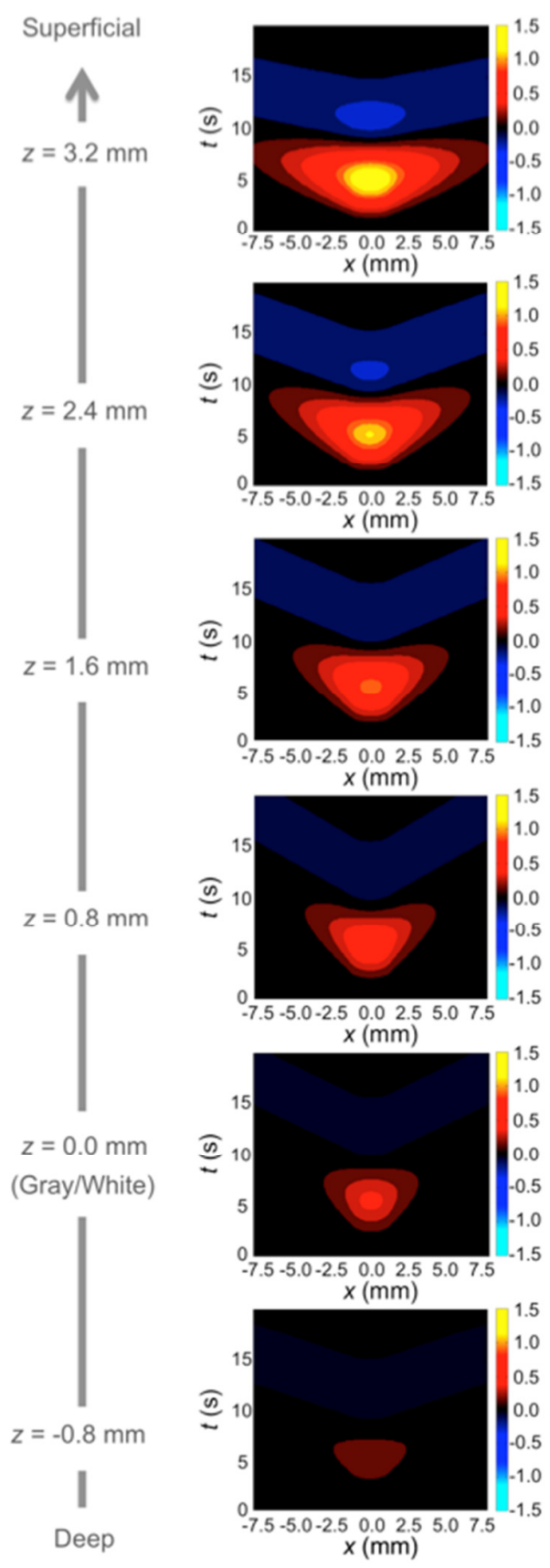

502 Figure 4. Modeled spatiotemporal HRF plots vs. cortical depth relative to the 503 gray/white boundary, $z$. These plots were constructed using the empirically 504 derived velocity, damping, and amplitude estimates (Fig. 3) and the theoretically 505 derived framework of the poroelastic model of hemodynamics (Aquino et al 506 2014). 


\subsection{Implications for hemodynamic modeling}

510 The spatiotemporal dynamics found in this study are consistent with a poroelastic

511 model of hemodynamics (Drysdale et al., 2010). In particular, hemodynamic

512 waves were observed and could be characterized with velocity and damping

513 coefficients which are within predicted ranges (Aquino et al. 2014). Although the

514 poroelastic model averages cortical vascular anatomy in a continuous mean field,

515 key dynamics are accurately represented and related to physiology thus enabling

516 it to be used to deconvolve BOLD signals to estimate the underlying neuronal

517 responses that drive the hemodynamic response.

519 The variation of the hemodynamic parameters versus cortical depth we show

520 provides insights and constraints into extending hemodynamic modeling into the

521 depth dimension. As indicated by previous vascular physiology, an important

522 consideration is to account for changes in cortical elasticity as a function of

523 depth. Including this in the poroelastic model can be accomplished by adding

524 heterogeneities in the model equations thus changing the dynamics as a function

525 of depth, namely the hemodynamic velocity. While the depth-dependent changes

526 in velocity observed here are consistent with known changes in vascular

527 elasticity, it is important to note that other vascular properties known to change

528 across cortical depth may also contribute, such as vascular density (Tsai et al., 529 2009). 


\subsection{Limitations and considerations}

532 Previous work has shown that the temporal properties of the HRF vary across a

533 number of factors such as cortical region (Handwerker et al., 2004; Puckett et al.,

534 2014; Soltysik et al., 2004). Since our model parameters were found by

535 measuring the response in primary visual cortex it remains unknown how

536 generalizable these parameters are to other cortical regions. Moreover, some

537 aspects of the HRF may depend on MRI acquisition parameters such as field

538 strength and sequence type (Davis et al., 1998; Obata et al., 2006; Siero et al.,

539 2013). It is important to note, however, that the technique we employ (gradient

540 echo at $3 \mathrm{~T}$ ) is the most commonly used today, and hence the data provided is

541 positioned to inform a large body of work.

543 At present there is still significant debate about the general feasibility of using

$544 \mathrm{fMRI}$ to infer depth-dependent neuronal activity. While in principle effects of

545 vessels from deeper cortical layers such as layer 4, passing through higher

546 cortical layers can be disentangled (Heinzle et al., 2016), the spatial spread of

547 vascular responses is unclear. Our study here addresses spatial spread

548 tangential to the cortical surface and how it changes with cortical depth, but we

549 cannot characterize spatial spread orthogonal to the cortical surface, through the

550 depth of cortical layers. As such, the across depth variation in the spatiotemporal

551 HRF is currently modeled in a stratified fashion, using a set of independent HRF

552 profiles describing the structure of the response tangential to the surface at 
553 different depths, z (Fig. 4). Future work is required to connect these profiles by

554 incorporating across layer dynamics into the model.

555

556 Using the HRF data provided here to estimate the underlying neuronal responses

557 also requires an assumption regarding the neural response profile elicited by our

558 stimulus. A uniform neural response profile across depth is the simplest

559 assumption, and the linear changes in the HRF we report are well explained

560 through a linear change in vascular properties, with no contribution from laminar

561 changes in neuronal activity. It has been shown in macaque that a simple visual

562 stimulus elicits uniform multi-unit spike activity across cortical depth (Xing et al.,

563 2012) suggesting this may be a reasonable assumption. However, the same

564 study revealed that local field potential activity showed a non-uniform response

565 profile across depth suggesting that some of the HRF variation we observe may

566 reflect non-uniformity in neuronal responses as suggested previously (Koopmans

567 et al., 2010).

568

569 Another important issue is the challenge of defining laminar surfaces using MRI

570 data, with two main automatic techniques in the literature: (i) equidistant models,

571 i.e. sampling layers by fraction of the cortical thickness (Muckli et al., 2015;

572 Olman et al., 2012; Polimeni et al., 2010) and (ii) equivolume techniques, i.e.,

573 sampling using Bok's principle (Waehnert et al., 2014). These methods take into

574 account the variation of thickness and curvature and normalize the surface

575 sampling methods. For our study we employed an absolute sampling method 
576 that ignores this variation. We note, however, that because the responses

577 reported here are from a single eccentricity in $\mathrm{V} 1$ and that the curvature and

578 thickness are uniform in most of V1 (Fischl \& Dale, 2000; Hinds et al., 2008) the

579 methods should yield equivalent results. It is also important to reiterate that the

580 most superficial laminar surface $(z=3.2 \mathrm{~mm})$ is likely in the vascular arachnoid,

581 outside of the cortical sheet. Although sampling outside of cortex, the BOLD

582 response measured here still reflects changes in neuronal activity as blood

583 vessels that feed/drain the responses within cortex exist in this range. These

584 responses are also important to consider as they are likely to further inform

585 mechanisms of spatial spreading (Gao et al., 2015; Kim et al., 2013). We

586 currently model this response (Fig. $4, z=3.2 \mathrm{~mm}$ ) using the same poroelastic

587 model derived for responses within the cortical sheet. In the context of the model,

588 the vessels in the arachnoid are connected to the pores within the cortical sheet.

589 This appears to yield a reasonable approximation of the empirical response (cf.

590 Fig. 2); however, future work will be needed to take into account differences in

591 the underlying physiology that exists between the vascular arachnoid and the

592 cortical sheet

593

\section{4.4 Conclusion}

595 The spatiotemporal BOLD response to a simple visual stimulus propagates from

596 its source (i.e., the neuronal drive) through the surrounding vasculature. We

597 report how fundamental properties of the response vary as a function of cortical

598 depth. By quantifying spatiotemporal properties of the HRF tangential and 
599 perpendicular to the cortical surface, we provide a characterization of an HRF 600 able to capture this variation, finding that the temporal and spatial responses are 601 fundamentally inseparable. Together with spatiotemporal deconvolution this lays 602 the foundation to infer depth-dependent neuronal activity, which importantly can 603 be related to cortical layers either through previous knowledge or careful co604 registration with high-resolution structural MRI data. 


\section{Acknowledgments}

606 We thank Zoey Isherwood, Ruth Png, and Wenbin Shao for technical

607 contributions. We also thank Dr. Josef Pfeuffer for developing and Siemens

608 Healthcare for providing the prototype $\mathrm{fMRI}$ sequence package used for data

609 acquisition. This research was supported by Australian Research Council (ARC)

610 grants DP120100614 and DP130100437, the Center of Excellence for Integrative

611 Brain Function (ARC Center of Excellent Grant CE140100007), the ARC

612 Laureate Fellowship Grant (FL140100025) and the NHMRC (APP). 
614 Aquino, K. M., Schira, M. M., Robinson, P. A., Drysdale, P. M. \& Breakspear, M. (2012). Hemodynamic traveling waves in human visual cortex. PLoS Comput Biol 8, e1002435.

617 Aquino, K. M., Robinson, P. A., Schira, M. M. \& Breakspear, M. (2014). 618 Deconvolution of neural dynamics from fMRI data using a spatiotemporal 619 hemodynamic response function. Neurolmage 94, 203-215, 620 doi:10.1016/j.neuroimage.2014.03.001.

621 Bastos, A. M., Usrey, W. M., Adams, R. A., Mangun, G. R., Fries, P., \& Friston, 622 K. J. (2012). Canonical microcircuits for predictive coding. Neuron 76, $623 \quad 695-711$.

624 Biessmann, F., Murayama, Y., Logothetis, N. K., Muller, K. R. \& Meinecke, F. C. 625 (2012). Improved decoding of neual activity from fMRI signals using nonseparable spatiotemporal deconvolutions. Neurolmage 61, 1031-1042.

627 Birn, R. M. \& Bandettini, P. A. (2005). The effect of stimulus duty cycle and "off" duration on BOLD response linearity. Neurolmage 27, 70-82.

629 Boynton, G. M., Engel, S. A., \& Heeger, D. J. (2012). Linear systems analysis of 630 the fMRI signal. Neurolmage 62, 975-984.

631 Brainard, D. H. (1997). The Psychophysics Toolbox. Spat Vis 10, 433-436.

632 Bronstein, A. M., Bronstein, M. M., \& Kimmel, R. (2008) Numerical Geometry of 633 Non-Rigid Shapes (Springer). 
634 Buxton, R. B., Wong, E. C., \& Frank, L. R. (1998). Dynamics of blood flow and oxygenation changes during brain activation: the balloon model. Magn Reson Med 39, 855-864.

637 Chen, G., Wang, F., Gore, J. C., \& Roe, A. W. (2013). Layer-specific BOLD activation in awake monkey $\mathrm{V} 1$ revealed by ultra-high spatial resolution functional magnetic resonance imaging. Neurolmage 64, 147-155.

640 Davis, T. L., Kwong, K. K., Weisskoff, R. M., \& Rosen, B. R. (1998). Calibrated functional MRI: mapping the dynamics of oxidative metabolism. Proceedings of the National Academy of Sciences, 95(4), 1834-1839.

DeYoe, E. A., Carman, G. J., Bandettini, P., Glickman, S., Wieser, J., Cox, R., Miller, D., \& Neitz, J. (1996). Mapping striate and extrastriate visual areas in human cerebral cortex. Proc Natl Acad Sci USA 93, 2382-2386.

Drysdale, P., Huber, J.P., Robinson, P.A., \& Aquino, K.M. (2010). Spatiotemporal BOLD hemodynamics from a poroelastic hemodynamic model. $\mathrm{J}$ Theor Biol 265, 524-534.

649 Duvernoy, H. M., Delon, S. \& Vannson, J. L. (1981). Cortical blood vessels of the human brain. Brain Res Bull 7, 519-579.

651 Engel, S. A., Glover, G. H., \& Wandell, B. A. (1997). Retinotopic organization in 652 human visual cortex and the spatial precision of functional MRI. Cerebral $653 \quad$ Cortex 7, 181-192.

654 Fischl, B. \& Dale, A. M. (2000). Measuring the thickness of human cerebral 655 cortex from magnetic resonance images. PNAS 97, 11050-11055. 
656 Friston, K. J., Jezzard, P., \& Turner, R. (1994). Analysis of functional MRI timeseries. Hum Brain Mapp 1, 153-171.

658 Friston, K.J., Mechelli, A., Turner, R., \& Price, C.J. (2000). Nonlinear responses in fMRI: the Balloon model, Volterra kernels, and other hemodynamics. Neurolmage 12, 466-477.

661 Gao, Y. R., Greene, S. E., \& Drew, P. J. (2015). Mechanical restriction of intracortical vessel dilation by brain tissue sculpts the hemodynamic response. Neurolmage 115, 162-176.

664 Gardner, J. L. (2010). Is cortical vasculature functionaly organized? Neurolmage 49, 1953-1956.

666 Griswold, M. A., Jakob, P. M., Heidemann, R. M., Nittka, M., Jellus, V., Wang, J., Kiefer, B., \& Haase, A. (2002). Generalized autocalibrating partially parallel acquisitions (GRAPPA). Magn Reson Med 47, 1202-1210, doi:10.1002/mrm.10171.

670 Haacke, E. M., Brown, R. W., Thompson, M. R., \& Venkatesan, R. (1999).

671 Magnetic Resonance Imaging: Physical Principles and Sequence Design.

$672 \quad$ (Wiley-Liss).

673 Handwerker, D. A., Ollinger, J. M., \& D’Esposito, M. (2004). Variation of BOLD 674 hemodynamic responses across subjects and brain regions and their effects 675 on statistical analyses. Neurolmage, 21(4), 1639-51. 676 doi:10.1016/j.neuroimage.2003.11.029 
677 Heinzle, J., Koopmans, P. J., den Ouden, H. E. M., Raman, S., \& Stephan, K. E. (2016). A hemodynamic model for layered BOLD signals. Neurolmage $125,556-570$.

680 ladecola, C. (2004). Neurovascular regulation in the normal brain and in Alzheimer's disease. Nat Rev Neurosci 5, 347-360.

682 Jin, T. \& Kim, S. G. (2008). Cortical layer-dependent dynamic blood oxygenation, cerebral blood flow and cerebral blood volume responses during visual stimulation. Neurolmage 43, 1-9.

685 Kim J. H., Khan R., Thompson J. K., \& Ress D. (2013) Model of the transient neurovascular response based on prompt arterial dilation. Journal of Cerebral Blood Flow and Metabolism: Official Journal of the International Society of Cerebral Blood Flow and Metabolism. 33, 1429-39. doi: 10.1038/jcbfm.2013.90

690 Koopmans, P. J., Barth, M. \& Norris, D. G. (2010). Layer-specific BOLD activation in human V1. Hum Brain Mapp 31, 1297-1304.

692 Kriegeskorte, N., Cusack, R., \& Bandettini, P. (2010). How does an fMRI voxel sample the neuronal activity pattern: Compact-kernel or complex spatiotemporal filter? Neurolmage 49, 1965-1976.

$695 \mathrm{Li}$, J., et al. (2012). Reducing the object orientation dependence of susceptibility 696 effects in gradient echo MRI through quantitative susceptibility mapping. 697 Magn Reson Med 68, 1563-1569.

698 Logothetis, N. K. (2008). What we can and what we can't do with fMRI. Nature 699 453, 869-878. 
700 Martin, C., Zheng, Y., Sibson, N. R., Mayhew, J. E. W., \& Berwick, J. (2013). Complex spatiotemporal haemodynamic response following sensory stimulation in the awake rat. Neurolmage, 66, 1-8.

703 Muckli, L., De Martino, F., Vizioli, L., Petro, L. S., Smith, F. W., Ugurbil, K., \& 704 Yacoub, E. (2015). Contextual Feedback to Superficial Layers of V1. Current Biology, 25(20), 2690-2695. doi:10.1016/j.cub.2015.08.057.

Obata, T., Liu, T. T., Miller, K. L., Luh, W., Wong, E. C., Frank, L. R., \& Buxton, R. B. (2004). Discrepancies between BOLD and flow dynamics in primary and supplementary motor areas: application of the balloon model to the interpretation of BOLD transients. Neurolmage 21(1), 144-53. doi:10.1016/j.neuroimage.2003.08. 040.

Olman, C. A., Harel, N., Feinberg, D.

D. A., He, S., Zhang, P., Ugurbil, K., \& Yacoub, E. (2012). Layer-specific fMRI reflects different neuronal

714 Pelli, D. G. (1997). The VideoToolbox software for visual psychophysics: transforming numbers into movies. Spat Vis 10, 437-442.

716 Polimeni, J. R., Fischl, B., Greve, D. N. \& Wald, L. L. (2010). Laminar analysis of 7 T BOLD using an imposed spatial activation pattern in human V1. Neurolmage 52, 1334-1346.

719 Puckett A. M., Mathis, J. R., \& DeYoe, E. A. (2014). An investigation of positive 720 and inverted hemodynamic response functions across multiple visual $721 \quad$ areas. Human Brain Mapping 35:5550 -5564. 
722 Ress, D., Glover, G. H., Liu, J. \& Wandell, B. (2007). Laminar profiles of 723 functional activity in the human brain. Neuroimage 34, 74-84.

724 Schira, M. M., Tyler, C. W., Breakspear, M., \& Spehar, B. (2009). The foveal 725 confluence in human visual cortex. J Neurosci 29, 9050-9058, doi:10.1523/JNEUROSCI.1760-09.2009.

Schira, M. M., Lei J., Yakupov, R., Kaule, F.K., Speck, O., Aquino, K. M., Isherwood, Z., Breakspear, M. J., \& Hoffmann, M. B. (2010). Sub millimeter resolution $\mathrm{fMRI}$ (both $3 \& 7 \mathrm{~T}$ ); BOLD modulation results in motion correction artifact. Presented at the Organization of Human Brain Mapping annual conference.

Schira, M. M., Tyler, C. W., Spehar, B., \& Breakspear, M. (2010). Modeling magnification and anisotropy in the primate foveal confluence. PLoS Comput Biol 6, e1000651.

Scholtens, L. H., Reus, M. A., \& van den Heuvel, M. P. (2015). Linking contemporary high resolution magnetic resonance imaging to the von economo legacy: A study on the comparison of MRI cortical thickness and histological measurements of cortical structure. Human Brain Mapping 36, 3038-3046.

Sethian, J. A. (1999). Level Set Methods and Fast Marching Methods Evolving Interfaces in Computational Geometry, Fluid Mechanics, Computer Vision, 744 and Materials Science (Cambridge University Press).

Shipp, S. (2007). Structure and function of cerebral cortex. Curr Biol 17, R443449. 
745 Shmuel, A., Yacoub, E., Chaimow, D., Logothetis, N. K., \& Ugurbil, K. (2007). Spatio-temporal point-spread function of $\mathrm{fMRI}$ signal in human gray matter at 7 Tesla. Neurolmage $35,539-552$.

748 Siero, J. C. W., Hendrikse, J., Hooduin, H., Petridou, N., Luijten, P., \& Donahue, M. J. (2015). Cortical depth dependence of the BOLD initial dip and poststimulus undershoot in human visual cortex at 7 Tesla. Magn Reson Med 73, 2283-2295.

Siero, J. C. W., Ramsey, N. F., Hoogduin, H., Klomp, D. W. J., Luijten, P. R., \& Petridou, N. (2013). BOLD specificity and dynamics evaluated in humans

Siero, J. C. W., Petridou, N., Hoogduin, H., Luijten P. R., \& Ramsey, N. F. (2011). Cortical depth-dependent temporal dynamics of the BOLD response in the human brain. JCBFM 31, 1999-2008.

759 Silva, A. C, Lee, S. (2000). Early temporal characterstics of cerebral blood flow and deoxyhemoglobin changes during somatosensory stimulation. JCBFM 20, 201-206.

762 Soltysik, D. A., Peck, K. K., White, K. D., Crosson, B., \& Briggs, R. W. (2004). Comparison of hemodynamic response nonlinearity across primary cortical areas. Neurolmage 22, 1117-1127.

Stephan, K.E., Weiskopf, N., Drysdale, P.M., Robinson, P.A., \& Friston, K.J. (2007). Comparing hemodynamic models with DCM. Neurolmage 38, 387-401. 
768 Tournier, JD., Calamante, F., Gadian, D.G., \& Connelly, A. (2004). Direct estimation of the fiber orientation density function from diffusion-weighted MRI data using spherical deconvolution. Neurolmage 23, 1176-1185.

771 Tsai, P. S., Kaufhold, J. P., Blinder P., Friedman, B., Drew, P. J., Karten, H. J., Lyden, P. D., \& Kleinfeld, D. (2009). Correlations of neuronal and microvascular densities in murine cortex revealed by direct counting and colocalization of nuclei and vessels. J Neurosci 29, 14554-14570.

775 Tootell, R. B., Silverman, M. S., \& De Valois, R. L. (1981). Spatial frequency 776 columns in primary visual cortex. Science 214, 813-5.

777 Vanduffel, W., Tootell, R. B., Schoups, A. A., \& Orban, G. A. (2002). The 778 organization of orientation selectivity throughout macaque visual cortex.

780 Van Essen, D. C., Drury, H. A., Dickson, J., Harwell, J., Hanlon, D., \& Anderson, 781 C. H. (2001). An integrated software suite for surface-based analyses of cerebral cortex. J Am Med Inform Assoc 8, 443-459.

783 Waehnert, M. D., Dinse, J., Weiss, M., Streicher, M. N., Waehnert, P., Geyer, S., 784 Turner, R., \& Basin, P.-L. (2014). Anatomically motivated modeling of cortical laminae. Neurolmage 93, 210-220.

786 Wagstyl, K., Ronan, L., Goodyer, I. M., \& Fletcher, P. C. (2015). Cortical thickness gradients in structural hierarchies. Neurolmage 111, 241-250.

788 Wandell, B. A. \& Wanawer, J. (2011). Imaging retinotopic maps in the human 789 brain. Vision Res 51, 718-737. 
790 Xing, D., Yeh, C., Burns, S., \& Shapley, R. M. (2012). Laminar analysis of 791 visually evoked activity in the primary visual cortex. Proc Natl Acad Sci U S A.109. 13871-13876.

793 Yu, X., Glen, D., Wang, S., Dodd, S., Hirano, Y., Saad, Z., Reynold, R., Silva, A. C., \& Koretsky, A. P. (2012). Direct imaging of macrovascular and microvascular contributions to BOLD fMRI in layer IV-V of the rat barrel cortex. Neurolmage 59, 1451-1460.

797 Yushkevich, P. A., Hazlett, H. C., Smith, R. G., Ho, S., Gee, J. C., \& Gerig, G. 798 (2006). User-guided 3D active contour segmentation of anatomical 799 structures: significantly improved efficiency and reliability. Neurolmage 31, $800 \quad$ 1116-1128, doi:10.1016/j.neuroimage.2006.01.015.

801 Zhang, E. T., Inman, C. B., \& Weller, R. O. (1990). Interrelationships of the pia 802 matter and the perivascular (Virchow-Robin) spaces in the human 803 cerebrum. J Anat 170, 111-123.

804 


\section{Supplementary Material:}

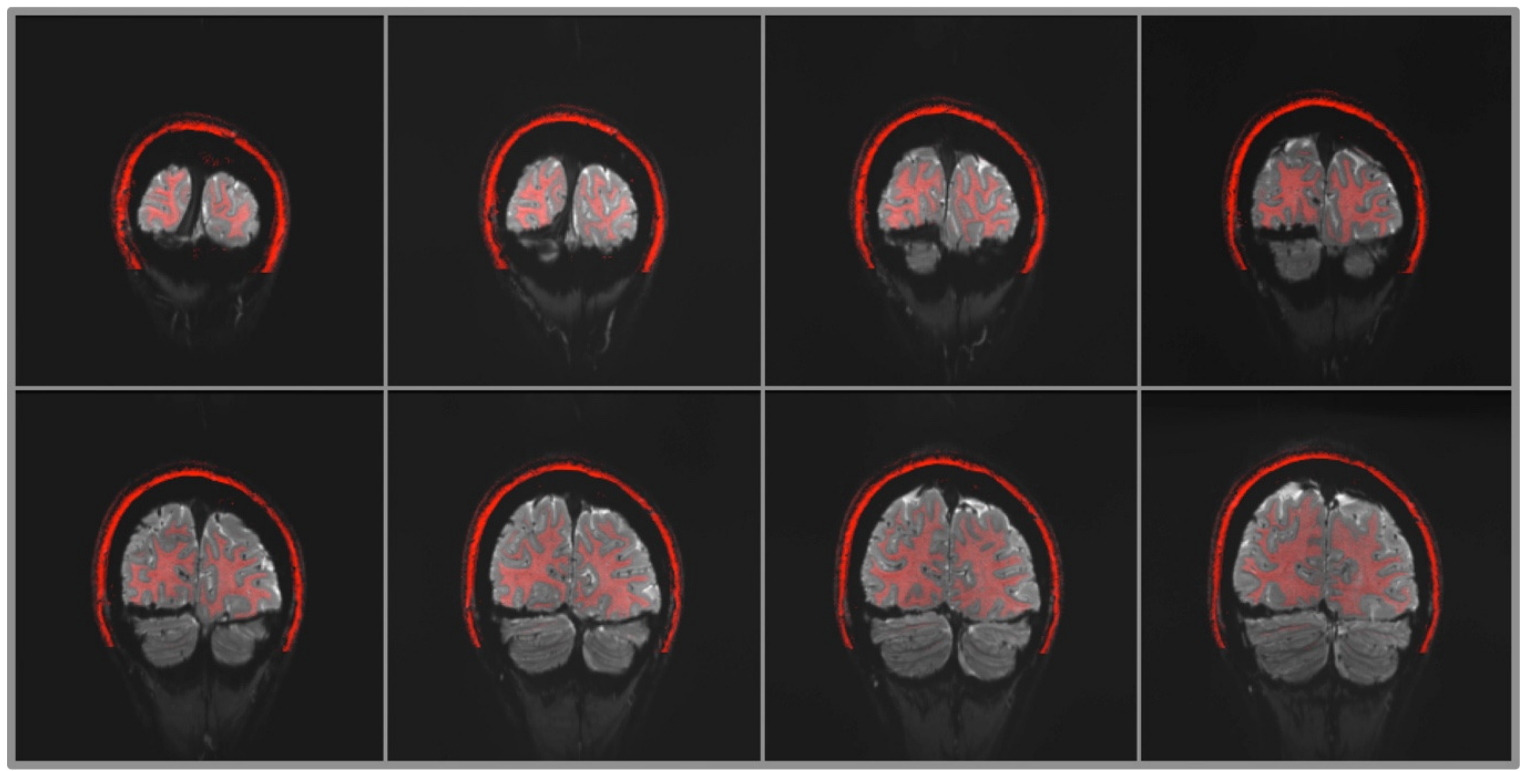

Figure S1: Example showing co-registration between the mean EPI image (gray) and the anatomical image (red). The anatomical image has been thresholded to emphasize white matter. Images are from a single subject and 8 slices are displayed.

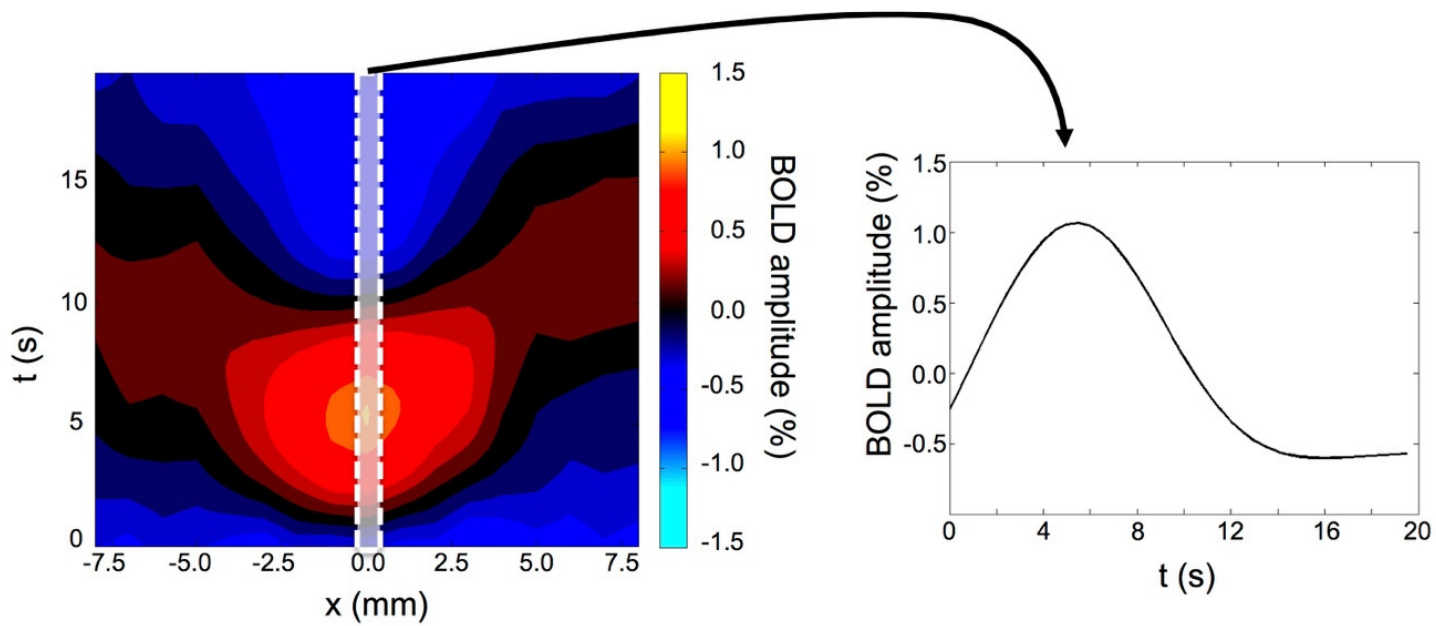

Figure S2: Illustration of the relationship between the spatiotemporal HRF profile (left) and a temporal HRF profile (right). The relationship may best be understood by considering the response only at the centerline (white rectangle, $x=0.0 \mathrm{~mm}$ ). If this response is plotted such that time is on the x-axis (rather than the $y$-axis) and amplitude is represented along the $y$-axis (rather than by color), then a temporal HRF profile is retrieved. The data used here were taken from the groupaveraged response at $z=1.6 \mathrm{~mm}$. The relationship between our spatiotemporal 
HRF and the temporal HRF is outlined mathematically and discussed in detail in previous publications (Aquino et al., 2012; Drysdale et al., 2010).

Video S3: Group-averaged spatiotemporal HRF video showing the structure tangential to the surface $(x)$ vs. space orthogonal to the surface $(z)$ with each frame representing the response at a different time point. Color indicates the percent modulation of BOLD.

Video S4: Single subject spatiotemporal HRF video showing the structure tangential to the surface $(x)$ vs. space orthogonal to the surface $(z)$ with each frame representing the response at a different time point. Color indicates the percent modulation of BOLD 